\section{Skeletal muscle cellularity and glycogen distribution in the hypermuscular Compact mice}

\author{
T. Kocsis, ${ }^{1}$ J. Baán, ${ }^{1}$ G. Müller, ${ }^{2}$ \\ L. Mendler, ${ }^{1}$ L. Dux, ${ }^{1}$ A. Keller-Pintér ${ }^{1}$ \\ 'Department of Biochemistry, Faculty \\ of General Medicine, University of Szeged \\ ${ }^{2}$ Egis Pharmaceuticals, Budapest, \\ Hungary
}

\section{Abstract}

The TGF-beta member myostatin acts as a negative regulator of skeletal muscle mass. The Compact mice were selected for high protein content and hypermuscularity, and carry a naturally occurring 12-bp deletion in the propeptide region of the myostatin precursor. We aimed to investigate the cellular characteristics and the glycogen distribution of the Compact tibialis anterior (TA) muscle by quantitative histochemistry and spectrophotometry. We have found that the deficiency in myostatin resulted in significantly increased weight of the investigated hindlimb muscles compared to wild type. Although the average glycogen content of the individual fibers kept unchanged, the total amount of glycogen in the Compact TA muscle increased two-fold, which can be explained by the presence of more fibers in Compact compared to wild type muscle. Moreover, the ratio of the most glycolytic IIB fibers significantly increased in the Compact TA muscle, of which glycogen content was the highest among the fast fibers. In summary, myostatin deficiency caused elevated amount of glycogen in the TA muscle but did not increase the glycogen content of the individual fibers despite the marked glycolytic shift observed in Compact mice.

\section{Introduction}

Myostatin (growth/differentiation factor-8, GDF-8), a member of the TGF-beta superfamily is a potent negative regulator of skeletal muscle growth. ${ }^{1}$ Knocking out of myostatin or naturally occurring mutations, e.g., in human, mice, cattle, dog and porcine ${ }^{2-6}$ lead to large and widespread increase of the skeletal muscle mass. Knockout mice show marked hypermuscularity due to both hyperplasia (increased fiber number) and hypertrophy (increased thickness of the fibers), ${ }_{1}^{1}$ whereas Belgian Blue double-muscled (DM) cattle contain almost double number of muscle fibers due to myostatin mutation. ${ }^{7,8}$ Muscle metabolism in DM cattle or knockout mice shifted into the glycolytic toward exhibiting an increased proportion of fast glycolytic white fibers. -10 $^{8-10}$

The Compact mice arose during a long-term selection program to reach the maximal protein accretion and hypermuscularity. ${ }^{11}$ Genetic analysis of the Hungarian subpopulation of the Compact line identified a 12-bp deletion in the prodomain region of the precursor myostatin; ${ }^{12}$ therefore, the biologically active growth factor part is intact. However, additional modifier genes should be present to determine the full expressivity of the phenotype. ${ }^{13,14}$ The precise biological, biochemical effect of the Compact mutation is poorly understood; the mutant propeptide region may play a role in the proper folding, secretion and targeting of mature myostatin. ${ }^{12}$ Hypermuscularity caused by Compact mutation results from muscle fiber hyperplasia rather than hypertrophy, and muscle metabolism shifted towards glycolytic direction. ${ }^{15,16}$ We hypothesized that not only the increased protein amount but also the higher glycogen content may account for the increased muscle weight of the Compacts; therefore, in this present study we aimed to analyze the muscle cellularity of the Compact mice focusing on the glycogen content and the fiber-type specific glycogen distribution. Here we show that the glycogen content of the IIB fibers was the highest among the fast fibers in the tibialis anterior (TA) muscle. The myostatin deficiency of Compact mice resulted in elevated glycogen content of the TA muscle but the average glycogen content of the individual fibers remained unchanged despite the marked glycolytic shift.

\section{Materials and Methods}

\section{Animals and sample collection}

This investigation conformed to the National Institutes of Health Guide for the Care and Use of Laboratory Animals (NIH Pub. No. 85-23, revised 1996) and was approved by the local ethics committee at the University of Szeged. We performed our experiments on 12 week-old male myostatin mutant Compact $(47.3 \pm 0.76 \mathrm{~g}$; $\mathrm{n}=8)$ and wild type BALB/c $(25.0 \pm 0.58 \mathrm{~g} ; \mathrm{n}=4)$ mice. The origin and the selection procedure of the Compact mice were described by Baán and colleagues. ${ }^{16}$ Briefly, the Compact line was selected initially in Berlin on the basis of high protein mass and hypermuscularity. The Hungarian subpopulation of this Compact line was inbred and kept for more than 20 years in the Institute for Animal Biology, Agricultural Animal Center (Gödöllő, Hungary), while they have been breeding since 2010 in the
Correspondence: Anikó Keller-Pintér, University of Szeged, Faculty of General Medicine, Department of Biochemistry, Dóm tér 9., H-6720 Szeged, Hungary.

Tel. 36.62.545097 - Fax: +36.62.545097.

E-mail: keller.aniko@med.u-szeged.hu

Key words: Compact mice, fiber-type, GDF-8, glycogen, muscle, myostatin.

Contributions: AKP, LM, GM, LD, study concept and design; TK, JB, data collection; TK, analysis and interpretation of data; TK, AKP, LM, manuscript drafting; AKP, LM, LD, critical revision of the manuscript; AKP, study supervision.

Acknowledgments: the authors would thank Zita Makráné Felhő and Istvánné Balásházi for their excellent technical assistance and Ernő Zádor and Mónika Kiricsi for the anti-MyHC antibodies.

Funding: this research was supported by the European Union and the State of Hungary, cofinanced by the European Social Fund in the framework of TÁMOP 4.2.4. A/2-11-1-2012-0001 National Excellence Program (to AKP), TÁMOP4.2.2.A-11/1/KONV-2012-0035 and Richter Gedeon Centenary Foundation.

Received for publication: 11 December 2013. Accepted for publication: 18 April 2014.

This work is licensed under a Creative Commons Attribution NonCommercial 3.0 License (CC BYNC 3.0).

(C) Copyright T. Kocsis et al., 2014

Licensee PAGEPress, Italy

European Journal of Histochemistry 2014; 58:2353 doi:10.4081/ejh.2014.2353

Department of Biochemistry, Faculty of General Medicine, University of Szeged (Szeged, Hungary). The non-selected BALB/c mice exhibiting wild-type myostatin gene were obtained from the Biological Research Centre of the Hungarian Academy of Sciences (Szeged, Hungary). The animals were kept under controlled temperature with $12 / 12 \mathrm{~h}$ light/dark cycles, and were fed standard chow and tap water ad libitum. Mice were anaesthetized by intraperitoneal injection of chloral hydrate (3\% chloral hydrate, $0.15 \mathrm{~mL} / 10 \mathrm{~g}$ body weight). The quadriceps femoris (QF), biceps femoris (BF), gastrocnemius (Gastro), tibialis anterior (TA) and extensor digitorum longus (EDL) muscles were removed and frozen immediately in isopentane cooled by liquid nitrogen and stored at $-80^{\circ} \mathrm{C}$ until further processing. The TA muscle from the right hindlimb was used for the spectrophotometric determination of glycogen and protein content, whereas morphological analysis [Periodic Acid Schiff (PAS)-staining, immunohistochemistry] was performed on the contralateral TA muscle. 


\section{Glycogen and protein determina- tion by spectrophotometry}

Muscle glycogen was measured as glucose residues after acidic hydrolysis by a standard enzymatic assay. Briefly, following cryogenic milling the samples were digested for $1.5 \mathrm{~h}$ in $2.0 \mathrm{M} \mathrm{HCl}(250 \mu \mathrm{L} \mathrm{HCl} / 10 \mathrm{mg}$ muscle tissue $)$ at $100^{\circ} \mathrm{C}$. After lysis the samples were cooled to room temperature and neutralized by adding of equal amount of $2.0 \mathrm{M} \mathrm{NaOH}$. Thereafter, the samples were centrifuged for $10 \mathrm{~min}$ at 21,000× g (Hettich Universal 320R, DJB, Labcare Ltd., Buckinghamshire, UK) at room temperature, and the supernatants were removed. The concentration of glucose was determined from the supernatant by Hexokinase kit (Roche, Mannheim, Germany).

Muscles were homogenized in a buffer $(0.1$ M Tris-HCl pH 8.0, 0.01 M EDTA, 10\% SDS) containing protease inhibitor cocktail (SigmaAldrich, St. Louis, MO, USA) to extract the total protein amount. The incubation of the samples at $99^{\circ} \mathrm{C}$ for $60 \mathrm{~s}$ was followed by centrifugation at $11,000 \times \mathrm{g}$ for $5 \mathrm{~min}$ (Hettich Universal 320R, DJB, Labcare Ltd.) at room temperature to remove cellular debris. The supernatant protein content was determined by BCA Protein Assay Reagent (Thermo Scientific, Rockford, IL, USA) in agreement with the manufacturer's instructions.

Spectrophotometry was performed on Fluostar Optima (BMG Labtech, Ortenberg, Germany) and data were analyzed on Mars Data Analysis Software (BMG Labtech).

\section{PAS-staining}

Glycogen was detected by performing PASstaining on $10 \mu \mathrm{m}$ cryosections of the TA muscle. Sections were fixed for $1 \mathrm{~h}$ at $4^{\circ} \mathrm{C}$ in $3.7 \%$ formaldehyde in ethanol immediately to avoid glycogen breakdown, and incubated for $15 \mathrm{~min}$ with $0.5 \%$ periodic acid (Sigma Aldrich) at room temperature, followed by $5 \mathrm{~s}$ washing in tap water and deionized water four times. Then the sections were incubated in Schiff's reagent for $1 \mathrm{~h}$ at room temperature, followed by 5 min incubation (twice) in potassium metabisulfite in deionized water. Thereafter, gently washing of the slides for $10 \mathrm{~s}$ under running hand-warm tap water was followed by washing three times for $5 \mathrm{~s}$ in deionized water. Finally, the sections were incubated for $30 \mathrm{~s}$ in $50,70,90,100 \%$ ethanol and for $15 \mathrm{~s}$ in toluol, then were mounted with Entellan.

\section{Immunohistochemistry}

Fiber-type analysis was performed on $10 \mu \mathrm{m}$ serial cryosections of the midbelly region of TA muscle. The sections were blocked in 5\% nonfat milk powder (BioRad, Berkeley, CA, USA) in PBS, and then incubated with mouse monoclonal primary antibodies. BA-D5 (1:25), sc-71 (1:25) and BFF3 (1:5) primary antibodies were used, specific for Myosin Heavy Chain I (MyHCI, slow oxidative), MyHCIIA (fast oxidative) and MyHCIIB (fast glycolytic). ${ }^{17,18}$ After incubation with the peroxidase-conjugated secondary antibody (rabbit anti-mouse; Dako, Glostrup, Denmark), the immunocomplexes were visualized by 3,3 '-diaminobenzidine. We could not detect BA-D5 positive (MyHCI) fibers in the TA muscle; therefore, the fibers stained with neither MyHCIIA nor MyHCIIB were considered as MyHCIIX fibers.

\section{Determination of the glycogen con- tent of the individual muscle fibers}

Photos were taken with 10x objective using a Nikon Labophot-2 microscope equipped with Olympus DP71 camera. The full cross sectional areas (CSAs) of the muscles were reconstructed from the microscopic images by Cell*B software (Olympus DP Soft software, ver. 3.2, Soft Imaging System $\mathrm{GmbH}$, Munster, Germany).

The glycogen content of an individual fiber $\left(\mathrm{OD}^{*} \mu \mathrm{m}^{2}\right)$ can be predicted by multiplying the average intensity of PAS-staining by the CSA of the fiber. Average intensity (optical density, OD) of PAS-staining (0-1 OD), CSAs of all fibers $\left(\mu \mathrm{m}^{2}\right)$ and fiber numbers were determined on the whole muscle CSA on greyscale converted PAS-stained, panoramic images by Digimizer software (Medcalc software, Mariakelke, Belgium).

The fiber-type specific average intensity of PAS-staining and the CSAs of the different fiber types were determined on 2-3 representative microscopic fields of both superficial and deep regions of the TA muscle (400-1000 fibers/sample, $14-17 \%$ of total fiber number with 10x objective). These muscle regions were selected for analysis because the tibialis anterior muscle shows an increasing gradient of fibres having a high oxidative enzyme activity proceeding from the superficial to the deep region of the muscle. ${ }^{19}$ Both the sc-71 (MyHCIIA) and the BF-F3 (MyHCIIB) stained regions were matched with the PAS-stained identical regions on serial sections to analyze the glycogen content of the different fiber types. The glycogen-index (\%) of a fiber-type was defined as the average $0 D^{*} \mu m^{2}$ value of a fiber type ${ }^{*}$ fiber type proportion / summarized $\mathrm{OD}^{*} \mu \mathrm{m}^{2 *}$ fiber type proportion values to compare the total glycogen content of the different fiber types.

\section{Statistical analysis}

Statistical evaluations were performed by unpaired $t$-test to assess the effect of Compact mutation on body weight, absolute and normalized weights of muscles, total protein and glycogen content of the whole TA muscle determined by spectrophotometry, relative distribution of the different fiber types, average inten- sity of PAS-staining of the fibers on whole muscle CSA, average area of the fibers, average glycogen content of the fibers and total glycogen content of the TA muscle based on PAS-staining. The applied statistical method was one-way ANOVA followed by NewmanKeuls post hoc test (GraphPad Software, Inc., San Diego, CA, USA) for the analysis of the fiber-type specific alterations of average area of fibers, average intensity of PAS-staining and average glycogen content of the different fiber types on the whole CSA or in the superficial and deep regions of TA muscle; and for the analysis of glycogen index. All data is expressed as means $\pm \mathrm{SEM}$.

\section{Results}

\section{Body weight and muscle weights}

First, we compared the body weights and the weights of the investigated hindlimb muscles of the Compact mice and the wild type BALB/c line. Here we show that the body weight of the Compact mice is almost two-fold larger $(\mathrm{P}<0.001$, Table 1). The absolute weights of the investigated hindlimb muscles such as quadriceps femoris, biceps femoris, gastrocnemius, tibialis anterior and extensor digitorum longus were at least two-fold larger in Compacts $(\mathrm{P}<0.001 ;$ Table 1). The muscle weights normalized to body weight significantly increased in Compact QF (1.23-fold; $\mathrm{P}<0.001)$, BF (1.3-fold; $\mathrm{P}<0.001)$, Gastro (1.34fold; $\mathrm{P}<0.001)$, TA $(1.35$-fold; $\mathrm{P}<0.001)$ and EDL $(1.3$-fold; $\mathrm{P}<0.01)$ muscles compared to $\mathrm{BALB} / \mathrm{c}$ line (Table 1).

\section{Muscle characteristics in Compact mice}

The Compact line was selected for high protein content and hypermuscularity. ${ }^{11}$ Beside the increased protein amount the higher glycogen content may account for the increased muscle weight of the Compact mice. Thus, we measured both total protein and glycogen amounts of the fast glycolytic TA muscle by spectrophotometry. Similarly to the two-fold increase in total protein content $(\mathrm{P}<0.001$; Table 1), the amount of glycogen was 1.9 -fold greater $(\mathrm{P}<0.01$; Table 1$)$ in the Compact $\mathrm{TA}$ muscle compared to the wild type. The remarkable increase in protein content was even higher than that of glycogen level proving the significance of protein accretion in the phenotype of Compact mice. Next we tested, whether the higher glycogen amount of the Compact muscle is caused by the changes in the glycogen content of the individual muscle fibers. To analyze this, first we determined the OD of PAS-staining and fiber sizes on the whole CSA of the TA muscle (Figure 1). The average 
intensity of PAS-staining did not show significant change in Compact muscle fibers compared to those of the wild type muscle (Figure 1C), and no significant difference was observed regarding the average CSAs of the fibers either (Figure 1D).

The average intensity of PAS-staining multiplied by the value of fiber size is suitable to estimate the average glycogen content of the individual fibers. Comparing the fibers average glycogen content we could not detect any significant changes between the two groups of animals (Figure 1E). Similar to previously reported results, ${ }^{15,16}$ the fiber number increased 1.7-fold in Compact animals $(\mathrm{P}<0.001$; Table 2). Multiplying the average glycogen content of the individual fibers by the increased fiber number we detected a similar, two-fold increase in the glycogen content of the whole muscle by PAS-staining $(\mathrm{P}<0.05$; Figure $1 F$ ) as measured by spectrophotometry (Table 1).

Taken together, the average glycogen content of the individual fibers did not change; therefore, the increased fiber number can explain the elevated glycogen amount of the Compact TA muscle.

\section{Fiber-type specific alterations in muscle cellularity}

To analyze the possible presence of the fiber-type specific alterations of the glycogen content, first we examined the fiber type composition of TA muscle and measured the CSAs of the different fiber types on serial sections immunostained by antibodies against the different isoforms of MyHCs (Figure 2). Regarding the fiber composition of the Compact muscle, we could not detect the slow MyHCI isoform in TA muscles (Table 2) in accordance with the literature. ${ }^{20}$ The ratio of IIB fibers $(\mathrm{P}<0.01$; Table 2$)$ increased 1.6-fold with concomitant decreases of IIX $(\mathrm{P}<0.05$; Table 2) and IIA fibers $(\mathrm{P}<0.001$; Table 2$)$ indicating a glycolytic shift. Because the number of IIA fibers in Compact TA was very low (Table 2), we could not perform statistical analysis on this type of fibers. Remarkable differences were observed comparing the sizes of the different fiber types on the whole CSA of the muscle. As in wild type animals, the CSA of type IIB fibers was 1.44-fold larger than those of type IIX; and IIX fibers were 1.52-fold larger than the IIA ones $(\mathrm{P}<0.01$; Figure $3 \mathrm{~A})$. In Compact mice we found a similar result: the CSA of type IIB fibers was 1.62-fold bigger than those of IIX fibers $(\mathrm{P}<0.001$; Figure 3A). Comparing the two mice lines we did not find any differences between the average size of either IIB or IIX fibers. The frequency distributions of both the IIX and IIB fiber sizes showed similar shapes in Compact and wild type mice (Figure $3 \mathrm{~B}, \mathrm{C}$ ). The histograms of IIX fibers (Figure $3 \mathrm{~B}$ ) revealed right-skewed distribution in both groups of animals. In case of IIB fibers the bellshaped curves were wider in Compact and BALB/c animals (Figure 3C) than those of IIX

fibers (Figure 3B). The superficial and deep regions of the TA muscle consist of different fiber types; ${ }^{10,19}$ therefore, we performed our measurements on both regions as well.

Table 1. Body weight, absolute and normalized weights of the quadriceps femoris, biceps femoris, gastrocnemius, tibialis anterior and extensor digitorum longus muscles of 12 week-old, male BALB/c and Compact mice $(n=4-8)$. Total protein amount and glycogen content of the TA muscle determined by spectrophotometry in BALB/c and Compact mice $(n=4-6)$.

\begin{tabular}{lrcc}
\hline Item & BALB/c & Compact & P value \\
Bw (g) & $25.0 \pm 0.58$ & $47.3 \pm 0.76$ & $<0.001$ \\
Muscle weights & & & \\
QF (mg) & $206 \pm 6.33$ & $470 \pm 12.19$ & $<0.001$ \\
BF (mg) & $167 \pm 7.23$ & $420 \pm 13.83$ & $<0.001$ \\
Gastro (mg) & $140 \pm 3.82$ & $352 \pm 7.42$ & $<0.001$ \\
TA (mg) & $45.3 \pm 0.73$ & $114 \pm 1.48$ & $<0.001$ \\
EDL (mg) & $10.0 \pm 0.65$ & $23.5 \pm 0.35$ & $<0.001$ \\
\hline Muscle weights/bw & & & \\
QF/bw (mg/g) & $8.0 \pm 0.12$ & $10.0 \pm 0.20$ & $<0.001$ \\
BF/bw (mg/g) & $6.5 \pm 0.12$ & $8.5 \pm 0.37$ & $<0.001$ \\
Gastro/bw (mg/g) & $5.5 \pm 0.11$ & $7.4 \pm 0.12$ & $<0.001$ \\
TA/bw (mg/g) & $1.77 \pm 0.03$ & $2.40 \pm 0.04$ & $<0.01$ \\
EDL/bw (mg/g) & $0.39 \pm 0.02$ & $0.51 \pm 0.01$ & $<0.001$ \\
Total protein amount in TA (mg) & $9.2 \pm 0.42$ & $18.9 \pm 0.38$ & $<0.01$ \\
\hline Total glycogen content in TA (mg) & $0.23 \pm 0.04$ & $0.44 \pm 0.02$ & \\
\hline
\end{tabular}

Bw, body weight; QF, M. quadriceps femoris; BF, M. biceps femoris; Gastro, M. gastrocnemius; TA, M. tibialis anterior; EDL, M. extensor digitorum longus. Values are mean $\pm \mathrm{SEM}$.

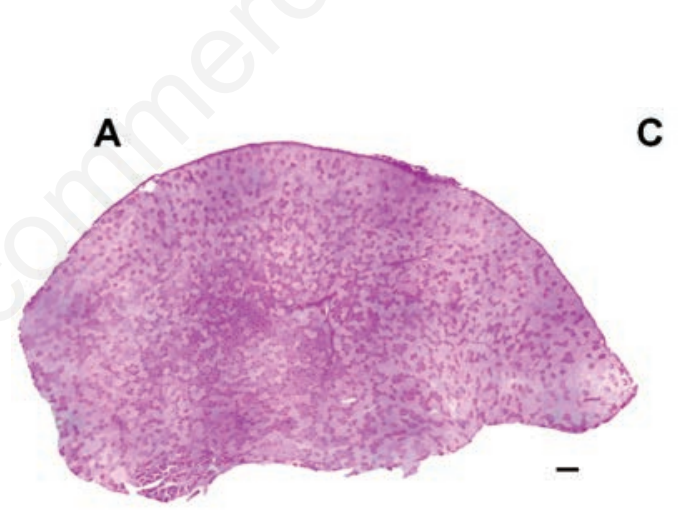

C $\quad E$
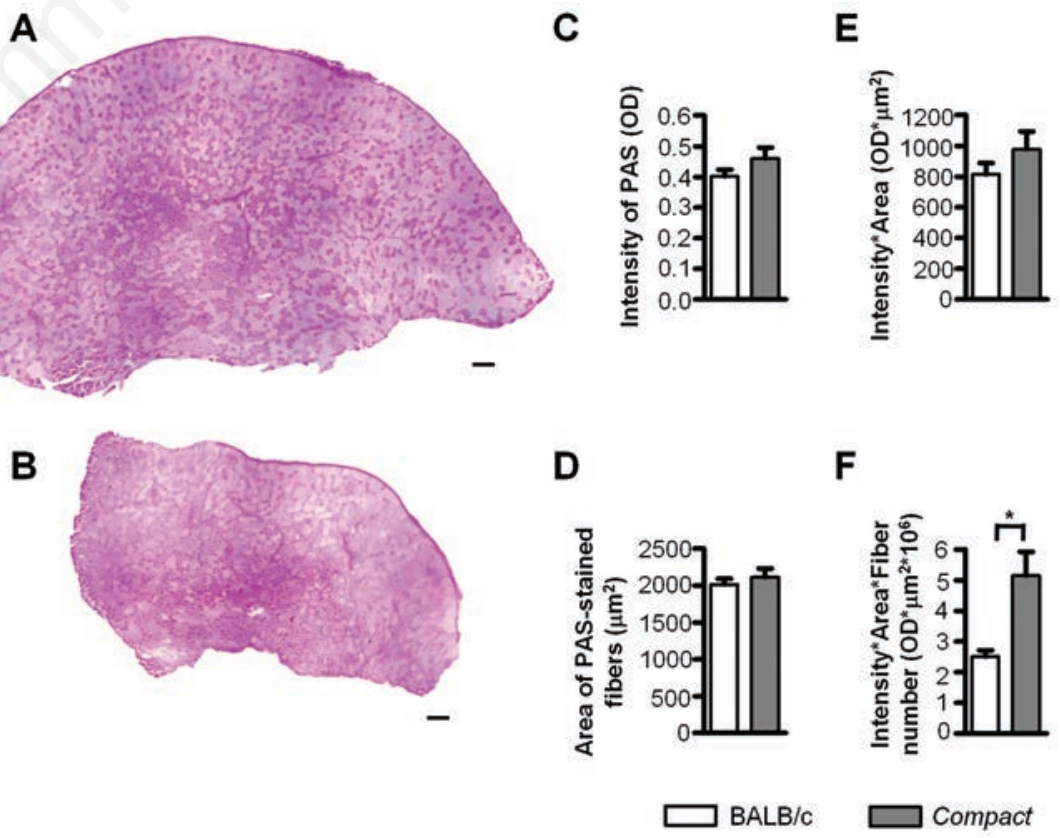

Figure 1. Representative panoramic images of Compact (A) and BALB/c (B) tibialis anterior muscles stained by PAS. Average intensity of PAS-staining of the fibers on whole muscle cross sectional area (C), average area of the fibers (D), average glycogen content of the individual fibers $(\mathrm{E})$ and total glycogen content of the tibialis anterior muscle $(\mathrm{F})$ based on PAS-staining $(n=3-3)$. Data are reported as mean $\pm S E M ;{ }^{* *} P<0.01 ;{ }^{* * *} P<0.001$. Scale bars: $200 \mu \mathrm{m}$. 
According to fiber size we found similar results, i.e., the area of IIB fibers was the greatest (IIA vs IIX, IIX $v s$ IIB, IIA $v s$ IIB in $\mathrm{BALB} / \mathrm{c}$ : superficial $\mathrm{P}<0.01$, deep: $\mathrm{P}<0.05$; IIX vs IIB in Compact: superficial $\mathrm{P}<0.001$; deep $\mathrm{P}<0.01$; Figure $4 \mathrm{~A}$ ), with no differences between the superficial and deep regions of the TA muscle in either mouse line, and no changes were observed regarding the fiber sizes of the two mice strains (Figure 4A).

To compare the effect of the Compact mutation on the fiber-type specific glycogen content of the muscle, we measured the average intensity of PAS-staining of the different fiber types on serial cryosections stained with antibodies against the different types of MyHC isoforms and PAS, respectively (Figure 2). The average intensity of PAS-staining showed a similar decreasing tendency in a rank order IIA $>$ IIX $>$ IIB on the whole CSA of the muscle: IIB fibers exhibited the weakest OD (Figure $3 \mathrm{~B})$, as in the superficial and deep regions (Figure 4B) in both mouse lines. The average intensity of PAS-staining of the different fiber types did not show significant differences between the Compact and BALB/c fibers on the whole muscle CSA (Figure 3D), and no significant changes could be observed comparing the PAS intensity of the fiber types in the superficial and deep regions either (Figure 4B). However, the frequency distribution revealed differences despite the similar average values of intensity of PAS-staining in Compact and wild type mice: the Compact TA muscle contained populations of IIX fibers with higher, and IIB fibers with both higher and lower intensity of PAS-staining resulting in a wider distribution in mutant animals (Figure 3 E,F). Next, the average glycogen content of the different fiber types was compared in both strains. The average glycogen content of the IIB fibers was greater than those of IIX fibers, whereas the IIA was the lowest in the superficial and deep regions of wild type muscle (Figure 4C). The results indicated significant difference in the superficial region of BALB/c animals (IIA $v s$ IIX, IIX $v s$ IIB, IIA $v s$ IIB; $\mathrm{P}<0.05$; Figure $4 \mathrm{C}$ ). The fiber type specific glycogen content on the whole CSA revealed similar tendency; however, without any significant changes (Figure 3G). In parallel with the frequency distribution of PAS intensity (Figure $3 \mathrm{E}, \mathrm{F})$, histograms of the average glycogen content (Figure $3 \mathrm{H}, \mathrm{I}$ ) showed that the Compact TA muscle contains a population of IIX fibers with higher, and IIB fibers with both higher and lower glycogen content .

Finally, we compared the total glycogen content of the different fiber types in the TA muscle. The glycogen index of IIB fibers was the highest, so that the IIB fibers stored the most glycogen in both BALB/c and Compact mice. The glycogen index value of IIX fibers was 2.7- fold larger $(\mathrm{P}<0.01)$ in $\mathrm{BALB} / \mathrm{c}$ mice compared to Compact animals (Figure 5) in line with the higher number of IIX fibers present in BALB/c mice. In contrast the glycogen index of IIB fibers was 1.5-fold bigger in Compacts $(\mathrm{P}<0.01)$ in good accordance with the increased proportion of IIB fibers in Compact mice.

\section{Discussion}

The Compact mutant mice carry a naturally occurring 12-bp non-frame shift deletion in the prodomain region of the precursor myostatin resulting hypermuscularity. ${ }^{12}$ Additional modifiers seem to be present to determine the full expression of the phenotype; however, these modifier genes have not been identified yet. ${ }^{13,14}$

The condition of the double muscled phenotype has been observed in several species. Natural mutations of myostatin can effect of the bioactive domain of myostatin: the mutation in Belgian Blue [nt821(del11)] causes a frame-shift virtually eliminating all of the mature, active regions of the molecule, whereas Piedmontese cattle presents a G938A transition resulting in a full-length, however misfolded and dysfunctional myostatin. ${ }^{4,21}$ Mutations in the pro-peptide leading an early

Table 2. Relative distribution of the different fiber types in BALB/c and Compact mice.

$\begin{array}{lccc}\text { Item } & \text { BALB } / \mathrm{c} & \text { Compact } & \text { P value } \\ \text { Total fiber number } & 3100 \pm 52.48 & 5200 \pm 227.6 & <0.001 \\ \text { Fiber type frequency (\%) } & & 0 & \\ \text { MyHCI } & 0 & 1 \pm 0.05 & <0.001 \\ \text { MyHCIIA } & 3 \pm 0.09 & 19 \pm 3.25 & <0.05 \\ \text { MyHCIIX } & 47 \pm 2.59 & 80 \pm 3.29 & <0.01 \\ \text { MyHCIIB } & 50 \pm 2.55 & \end{array}$

MyHC, Myosin Heavy Chain. Values are mean \pm SEM; $n=3-3$.
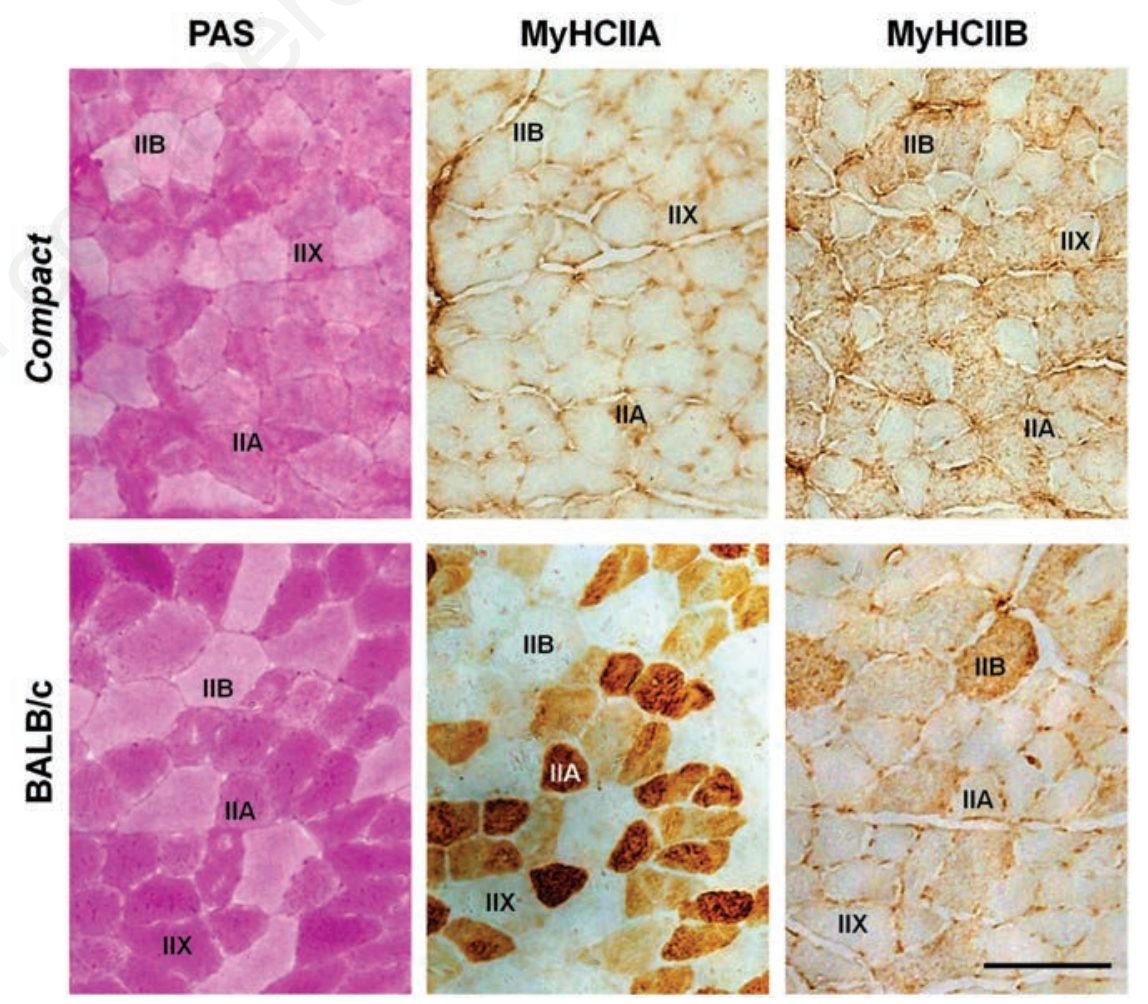

Figure 2. Representative microscopic images of the deep region of tibialis anterior muscle of BALB/c and Compact mice. Serial sections were stained by either PAS or antibodies against MyHCIIA and MyHCIIB isoforms. Representative fibers are marked as IIA, IIB and IIX. Scale bar: $100 \mu \mathrm{m}$. 
STOP codon [nt419(del7-ins10), Q204X, and E226X] have also been identified. ${ }^{22}$ The biologically active growth factor domain of myostatin is unaffected by Compact mutation, therefore the loss of myostatin activity cannot be explained by disruption of the growth factor bioactive domain; however, the Compact mutation can lead to misfolding, or defect in secretion and mistargeting of mature myostatin. ${ }^{2}$

In the present study we depict, similarly to previous observations, ${ }^{15,16,23,24}$ that the body and muscle weights of Compact animals increased compared to a mice line exhibiting wild type myostatin gene. Thus, the disproportionate increase of muscle weight can lead to increased body weight of Compact mice. Originally the Compact line was selected for hypermuscularity and high protein content at the Technical University in Berlin. ${ }^{11}$ Rehfeldt and colleagues introgressed the Compact mutation into the DUHi mice line. ${ }^{15}$ The hypermuscularity of this Compact-DUHi mice was characterized by muscle fiber hyperplasia rather than hypertrophy in fast glycolytic longissimus dorsi and rectus femoris muscles, and by balanced increases in myonuclear proliferation and protein accretion. ${ }^{15}$ However, no reports have so far examined the glycogen amount of the Compact muscles and the glycogen content of the individual fiber types. Here we show that the total glycogen amount of the TA muscle is markedly greater in Compact than in wild type mice. The phenotype selection on extreme muscle mass in DU6P mice

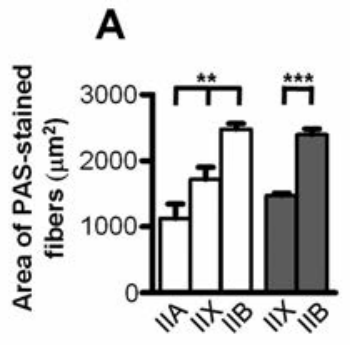

B

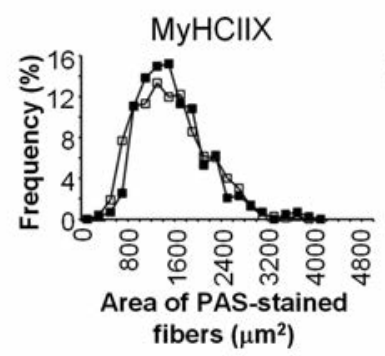

E
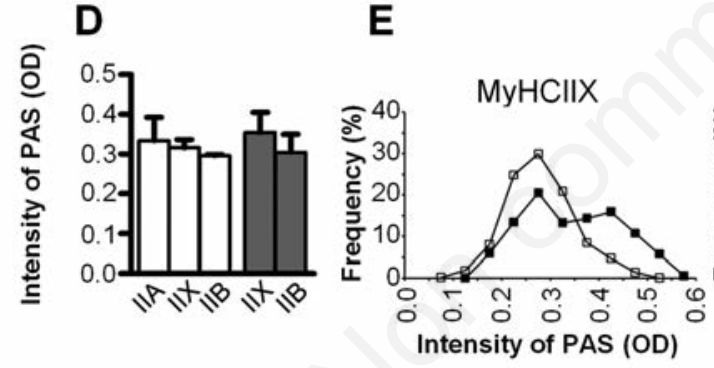

H
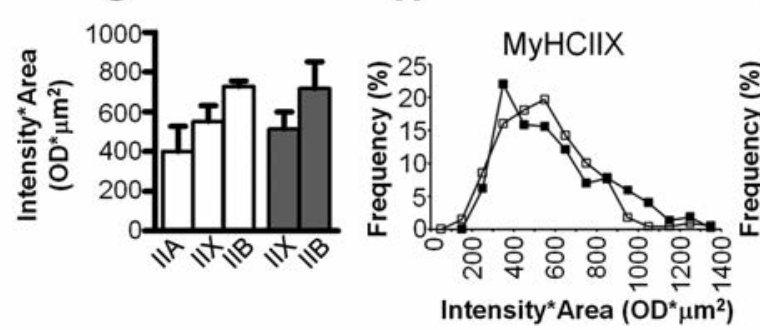

I
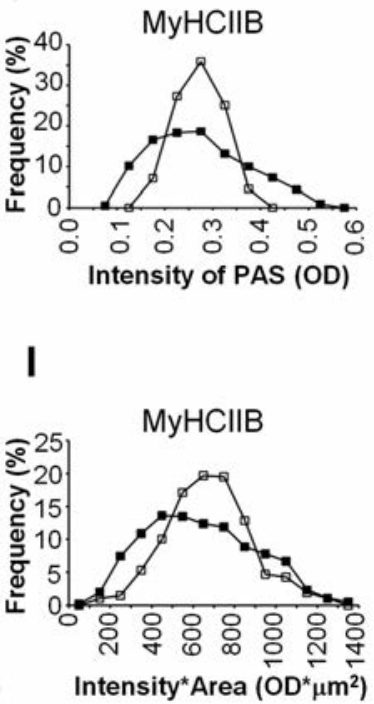

BALB/c

$\square$ Compact

๑ BALB/c

Compact

Figure 3. Average area of fibers (A), average intensity of PAS-staining (D) and average glycogen content of the different fiber types (G) on the whole cross sectional area of the tibialis anterior muscle in the Compact and BALB/c mice $(n=3-3)$. Histograms $(\mathrm{B}, \mathrm{C}, \mathrm{E}, \mathrm{F}, \mathrm{H}, \mathrm{I})$ show the frequency distribution of the defined parameters. Data are reported as mean $\pm \mathrm{SEM} ;{ }^{* *} \mathrm{P}<0.01 ;{ }^{* * *} \mathrm{P}<0.001$. revealed the co-evolution of high glycogen and protein content of the muscle. ${ }^{25}$ This finding is in line with our observation on the simultaneous increase of protein and glycogen contents in Compact muscle. The PI3K/Akt signaling plays a central role in integrating anabolic and catabolic responses in skeletal muscle influencing muscle mass. ${ }^{26,27}$ PTEN (Phosphatase and tensin homolog) negatively affects the

A

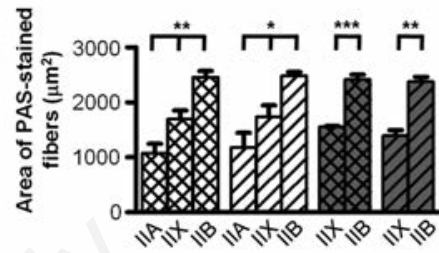

B

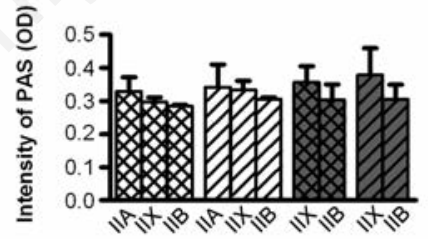

C

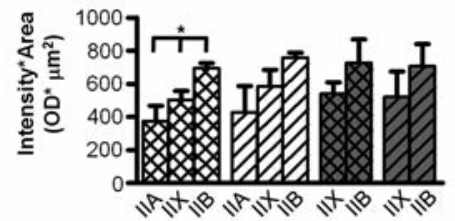

BALB/c superficial

Compact superficial

Z7] BALB/c deep

ZZ Compact deep

Figure 4. Average area of fibers (A), average intensity of PAS-staining (B) and average glycogen content of the fiber types $(C)$ in the superficial and deep regions of the tibialis anterior muscle in Compact and BALB/c mice $(n=3-3)$. Data are reported as mean \pm SEM; ${ }^{*} \mathbf{P}<0.05 ;{ }^{* *} \mathbf{P}<0.01 ;{ }^{* * *} \mathbf{P}<0.001$.

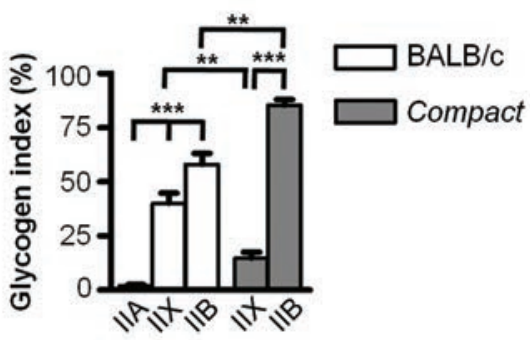

Figure 5. Glycogen index of the different (IIA, IIX and IIB) fiber types of the tibialis anterior muscle in $\mathrm{BALB} / \mathrm{c}$ and Compact mice. Data are reported as mean \pm SEM $\left(\mathbf{n}=3-3,{ }^{* *} \mathbf{P}<0.01{ }^{* * *} \mathbf{P}<0.001\right)$. 
PI3K/Akt pathway, ${ }^{28}$ moreover, Sawitzky and colleagues $^{25}$ identified PTEN as a gate keeper molecule in the co-evolutionary regulation of high glycogen and protein content.

In order to analyze the glycogen content of the individual muscle fibers the intensity of PAS-staining was measured. However, the value of the average $\mathrm{OD}$ of the pixels within a fiber cannot reflect the glycogen content of the whole muscle fiber; the OD value should be multiplied by the fiber size. In accordance with the literature ${ }^{15}$ we did not find differences comparing the fiber sizes of the Compact and wild type TA muscles. Furthermore, based on our results, the average glycogen content of the muscle fibers remained unchanged in Compacts compared to wild type mice; therefore, the increased number of fibers can explain the higher total glycogen amount of the Compact TA muscle. In addition to controlling muscle mass, myostatin also appears to regulate muscle fiber type composition postnatally by regulating the expression of both MyoD and MEF2C genes. ${ }^{29}$ Histological analysis revealed increased proportion of type IIB fibers in tibialis anterior, biceps femoris ${ }^{29}$ and EDL muscles $^{23}$ of myostatin knockout mice, or in double-muscled cattle. ${ }^{9,10}$ In accordance with our observations, earlier studies described that the Compact mutation induces a glycolytic shift in the phenotype of skeletal muscle, ${ }^{15,16}$ similarly to the situations evoked by the lack or the dysfunction of myostatin. Rehfeldt and her group reported that the number of white and intermediate fibers increased in rectus femoris and longissimus dorsi muscles of 70-day-old Compact mice, whereas the density of the blood capillaries decreased..$^{15}$ Despite the fact that the Compact mice are hypermuscular and their muscles consist of significantly more fibers, the specific force generation decreased in Compact animals. ${ }^{23}$ According to our results, the proportion of fast glycolytic fibers in TA muscle of Compact mice increased; however, their individual glycogen content did not.

Regarding the size of the different fiber types we found similar results in both Compact and $\mathrm{BALB} / \mathrm{c}$ mice: it increased in the rank order IIA $<$ IIX $<$ IIB among the fast fibers. In accordance with our observations, the area of IIB fibers was the biggest in the rectus femoris muscle of Compact-DUHi mice. ${ }^{15}$ The average glycogen content of the individual muscle fibers showed similar tendency as the fiber size (i.e., increased in the rank order IIA $<$ IIX $<$ IIB). Thus, the IIB fibers contained the most glycogen in both Compact and BALB/c mice, correlating with the biochemical properties of the fibers. Comparing the superficial and deep regions of the TA muscle we found similar results according to the fiber size, intensity of PAS-staining, as well as the glycogen content of the fibers. It is important to note that the measurement of the PAS intensity alone cannot predict clearly the glycogen content of the fibers (i.e., type IIB fibers have lower average PAS intensity but higher fiber size leading to larger glycogen content than that of IIX fibers). Only a few data are available on the glycogen content of the different types of fast fibers, mainly reported in human muscle biopsies. In accordance with our results, type IIA fibers showed more intense PAS-staining than IIB fibers in human biopsy samples. ${ }^{30}$

In conclusion, the phenotype of the Compact muscle is shifted towards glycolysis, similarly to previous observations. ${ }^{15,16}$ Among the fibers of the TA muscle, IIB fibers contained the most glycogen, accompanied by their increased number in Compact muscle. The myostatin mutation was associated with elevated glycogen content in the TA muscle without any change in the glycogen content of individual fibers despite the marked glycolytic shift.

\section{References}

1. McPherron AC, Lawler AM, Lee SJ. Regulation of skeletal muscle mass in mice by a new TGF- $\beta$ superfamily member. Nature 1997;387:83-90.

2. Schuelke M, Wagner KR, Stolz LE, Hübner C, Riebel T, Kömen W, et al. Myostatin mutation associated with gross muscle hypertrophy in a child. N Engl $\mathrm{J}$ Med 2004;350:2682-8.

3. McPherron AC, Lee SJ. Double muscling in cattle due to mutations in the myostatin gene. Proc Natl Acad Sci USA 1997;94: 12457-61.

4. Kambadur R, Sharma M, Smith TP, Bass JJ. Mutations in myostatin (GDF8) in double-muscled Belgian Blue and Piedmontese cattle. Genome Res 1997;7: 910-6.

5. Shelton GD, Engvall E. Gross muscle hypertrophy in whippet dogs is cause by a mutation in the myostatin gene. Neuromuscul Disorders 2007;17:721-2.

6. Stinckens A, Luyten T, Bijttebier J, Van den Maagdenberg K, Dieltiens D, Janssens S, et al. N. Characterization of the complete porcine MSTN gene and expression levels in pig breeds differing in muscularity. Anim Genet 2008;39:586-96.

7. Shahin KA, Berg RT. Growth patterns of muscle, fat and bone and carcass composition of double muscled and normal cattle. Can J Anim Sci 1985;65:279-93.

8. Wegner J, Albrecht E, Fiedler I, Teuscher F, Papstein HJ, Ender K. Growth and breedrelated changes of muscle-fiber characteristics in cattle. J Anim Sci 2000;78:1485-96.

9. Holmes JH, Ashmore CR. A histochemical study of development of muscle fibre type and size in normal and double muscled cattle. Growth 1972;36:351-72.

10. Matsakas A, Mouisel E, Amthor H, Patel K. Myostatin knockout mice increase oxidative muscle phenotype as an adaptive response to exercise. J Muscle Res Cell Motil 2010;31:111-25.

11. Bünger L, Laidlaw A, Bulfield G, Eisen EJ, Medrano JF, Bradford GE, et al. Inbred lines of mice derived from long-term on growth selected lines unique resources for mapping growth genes. Mamm Genome 2001;12:678-86.

12. Szabó G, Dallmann G, Müller G, Patthy L, Soller M, Varga L. A deletion in the myostatin gene causes the compact (Cmpt) hypermuscular mutation in mice. Mamm Genome 1998;9:671-2.

13. Varga L, Müller G, Szabó G, Pinke 0, Korom E, Kovács B, et al. Mapping modifers affecting muscularity of the myostatin mutant (MstnCmpt-dl1Abc) compact mouse. Genetics 2003;165:257-67.

14. Varga L, Pinke 0, Müller G, Kovács B, Korom E, Szabó G, et al. Mapping a syntenic modifier on mouse chromosome 1 influencing the expressivity of the compact phenotype in the myostatin mutant (MstnCmpt-dl1Abc) Compact mouse. Genetics 2005;169:489-93.

15. Rehfeldt C, Ott G, Gerrard DE, Varga L, Schlote W, Williams JL, et al. Effects of the Compact mutant myostatin allele Mstn(Cmpt-dl1Abc) introgressed into a high growth mouse line on skeletal muscle cellularity. J Muscle Res Cell Motil 2005;26:103-112.

16. Baán JA, Kocsis T, Keller-Pintér A, Müller G, Zádor E, Dux L, et al. The Compact mutation of myostatin causes a glycolytic shift in the phenotype of fast skeletal muscles. J Histochem Cytochem 2013;1:889-900.

17. Smerdu V, Soukup T. Demonstration of myosin heavy chain isoforms in rat and humans: the specificity of seven available monoclonal antibodies used in immunohistochemical and immunoblotting methods. Eur J Histochem 2008:52:179-90.

18. Schiaffino S, Gorza L, Sartore S, Saggin L, Ausoni S, Vianello M, et al. Three myosin heavy chain isoforms in type 2 skeletal muscle fibers. J Muscle Res Cell Motil 1989:10:197-205.

19. Ishihara A, Hirofuji C, Nakatani T, Itoh K, Itoh M, Katsuta S. Effects of running exercise with increasing loads on TA muscle fibres in mice. Exp Physiol 2002;87:113-6.

20. Carlson CJ, Booth FW, Gordon SE. Skeletal muscle myostatin mRNA expression is fiber-type specific and increases during hindlimb unloading. Am J Physiol Regul Integr Comp Physiol 1999;277:R601-6.

21. Berry C, Thomas M, Langley B, Sharma M, 
Kambadur R. Single cysteine to tyrosine transition inactivates the growth inhibitory function of Piedmontese myostatin. Am J Physiol Cell Physiol 2002;283:C135-41.

22. Grobet L, Poncelet D, Royo LJ, Brouwers B, Pirottin D, Michaux C, et al. Molecular definition of an allelic series of mutations disrupting the myostatin function and causing double-muscling in cattle. Mamm Genome 1998;9:210-3.

23. Amthor H, Macharia R, Navarrete R, Schuelke M, Brown SC, Otto A, et al. Lack of myostatin results in excessive muscle growth but impaired force generation. Proc Natl Acad Sci USA 2007;104:1835-40.

24. Amthor H, Otto A, Vulin A, Rochat A, Dumonceaux J, Garcia L, et al. Muscle hypertrophy driven by myostatin blockade does not require stem/precursor-cell activity. Proc Natl Acad Sci USA 2009;106:7479-84.

25. Sawitzky M, Zeissler A, Langhammer M, Bielohuby M, Stock P, Hammon HM, et al. Phenotype selection reveals coevolution of muscle glycogen and protein and PTEN as a gate keeper for the accretion of muscle mass in adult female mice. Plos One 2012;7:e39711.

26. Chelh I, Meunier B, Picard B, Reecy MJ, Chevalier C, Hocquette JF, et al. Molecular profiles of Quadriceps muscle in myostatin-null mice reveal PI3K and apoptotic pathways as myostatin targets. BMC Genomics 2009;10:196.

27. Wu M, Falasca M, Blough ER. Akt/protein kinase B in skeletal muscle physiology and pathology. J Cell Physiol 2011;226:29-36.

28. Schiaffino S, Dyar KA, Ciciliot S, Blaauw B, Sandri M. Mechanisms regulating skeletal muscle growth and atrophy. FEBS J 2013;280:4294-314.

29. Hennerby A, Berry C, Siriett V, 0'Callaghan P, Chau L, Watson T, et al. Myostatin regulates fiber-type composition of skeletal muscle by regulating MEF2 and MyoD gene expression. Am J Physiol Cell Physiol 2009;296:C525-34.

30. Dubowitz VC, Sewry A, Oldfors A. Normal muscle, p. 28-55. In: VC Dubowitz, A Sewry and A Oldfors (eds.) Muscle biopsy. A practical approach, 4th ed. Saunders Elsevier; 2013. 